\title{
Factors affecting curved versus straight path heading perception
}

\author{
CONSTANCE S. ROYDEN, JAMES M. CAHILL, and DANIEL M. CONTI \\ College of the Holy Cross, Worcester, Massachusetts
}

\begin{abstract}
Displays commonly used for testing heading judgments in the presence of rotations are ambiguous to observers. They can be interpreted equally well as motion in a straight line while rotating the eyes or as motion on a curved path. This has led to conflicting results from studies that use these displays. In this study, we tested several factors that might influence which of these two interpretations observers see. These factors included the size of the field of view, the duration of the stimulus, textured scenes versus random-dot displays, and whether or not observers were given a description of their path. The only factor that had a significant effect on path perception was whether or not observers were given instructions describing their path of motion. Under all conditions without instructions, we found that observers responded in a way that was consistent with the perception of motion on a curved path.
\end{abstract}

When an observer moves through the world, he or she must be able to judge his or her path of motion when moving in a straight line while making an eye rotation or when moving on a curved path. Previous research has yielded conflicting results about how this may be accomplished, with some results suggesting that extraretinal information is required for computing heading in the presence of eye movements (Banks, Ehrlich, Backus, \& Crowell, 1996; Ehrlich, Beck, Crowell, Freeman, \& Banks, 1998; Royden, Banks, \& Crowell, 1992; Royden, Crowell, \& Banks, 1994) and other results suggesting that this computation can be accomplished from the retinal information alone (Cutting, Springer, Braren, \& Johnson, 1992; Stone \& Perrone, 1997; van den Berg, 1992, 1993, 1996; Warren \& Hannon, 1988, 1990). Here, we examine some of the features of the stimuli commonly used in these experiments to help determine the reasons for these discrepancies.

When an observer moves in a straight line through a stationary scene, the image motion on the retina, known as the optic flow field, forms a radial pattern (Figure 1A) (Gibson, 1950, 1966; Longuet-Higgins \& Prazdny, 1980). The center of this pattern, known as the focus of expansion, corresponds to the observer's direction of motion. If the observer is undergoing a rotation while he or she is translating, as might occur if he or she were making an eye movement or were moving on a curved path, then the flow field is more complex (Figure 1B). Numerous models have been proposed to explain how the human visual system might recover the observer's motion parameters, both translation and rotation, from the flow field shown in Figure 1B (Beintema \&

We thank William Warren for helpful discussions on this work. This work was funded by NSF Grant IBN-0196068. Correspondence concerning this article should be sent to C. S. Royden, Department of Mathematics and Computer Sciences, P.O. Box 116A, College of the Holy Cross, Worcester, MA 01610 (e-mail: croyden@mathcs.holycross.edu). van den Berg, 1998; Hatsopoulos \& Warren, 1991; Heeger \& Jepson, 1992; Hildreth, 1992; Lappe \& Rauschecker, 1993; Perrone, 1992; Perrone \& Stone, 1994; Rieger \& Lawton, 1985; Royden, 1997). These models clearly show that it is possible to recover both translation (straight path motion) and rotation parameters from the optic flow field, but there has been considerable controversy in the literature about whether the human visual system can actually recover the translational component of motion separate from rotational motion using visual information alone. Some psychophysical results suggest that extraretinal information about eye movements is necessary for human observers to judge their translation direction accurately in the presence of eye movements (Banks et al., 1996; Ehrlich et al., 1998; Royden et al., 1992; Royden et al., 1994), whereas other results seem to suggest that visual information alone is sufficient (van den Berg, 1992, 1993, 1996; Warren \& Hannon, 1988, 1990).

Many of the experiments that measured these judgments used a design in which subjects observed two different types of displays: the "real" and "simulated" eye-movement conditions (Banks et al., 1996; Ehrlich et al., 1998; Regan \& Beverley, 1982; Rieger \& Toet, 1985; Royden et al., 1992; Royden et al., 1994; Warren \& Hannon, 1988, 1990). In the real eye-movement condition, the displays simulated motion in a straight line while observers tracked a moving fixation point. The rotation was generated by the tracking motion of the eyes. In the simulated eye-movement condition, the display simulated straight line motion with an eye movement, and observers fixated a stationary point on the screen. Thus, the stimulus on the retina in the two displays was identical, but in the real eye-movement condition, observers were actually moving their eyes, whereas in the simulated eye-movement condition, they were not. Although observers consistently judge the simulated heading quite well when viewing the real eye-movement condition (Banks et al., 1996; Ehrlich et al., 1998; Roy- 
A

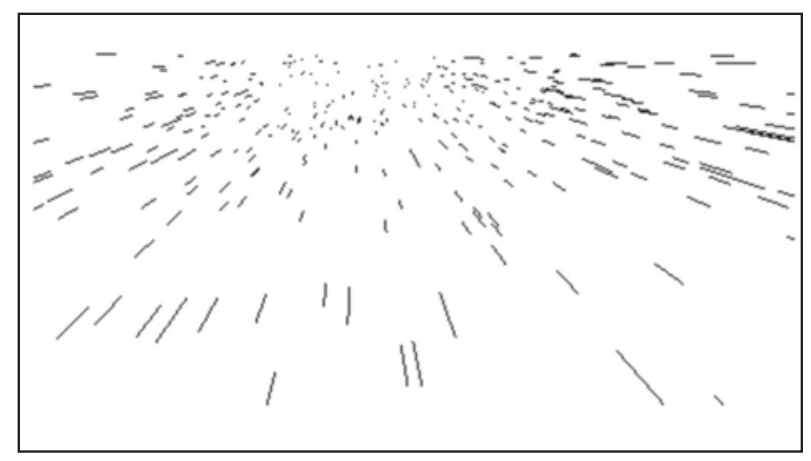

B

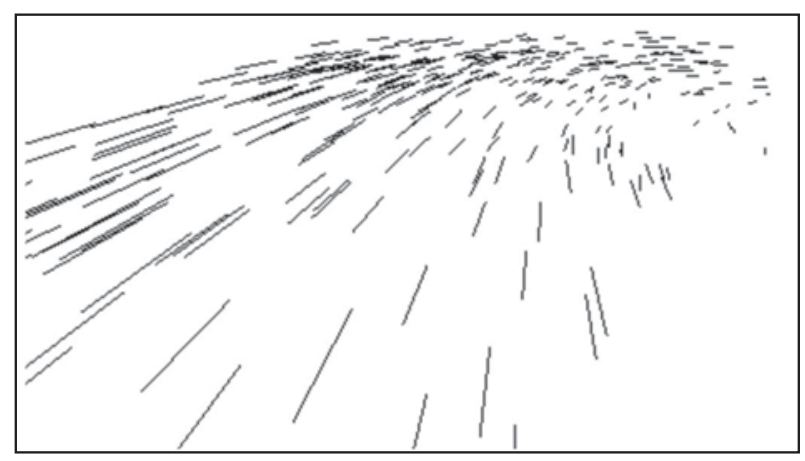

Figure 1. Optic flow fields. Each line indicates the speed and direction of an image point for an observer moving over a textured ground plane. (A) The radial optic flow pattern generated by an observer moving in a straight line. (B) The optic flow pattern for an observer moving with both translation and rotation - for example, walking in a straight line while making an eye or head movement.

den et al., 1992; Royden et al., 1994; Warren \& Hannon, $1988,1990)$, the results for the simulated condition vary in different studies, with some experiments yielding accurate results (van den Berg, 1992, 1993, 1996; Warren \& Hannon, 1988, 1990) and others resulting in rather large errors (Banks et al., 1996; Ehrlich et al., 1998; Royden et al., 1992; Royden et al., 1994). Royden (1994) noted that the simulated eye-movement condition is ambiguous, since it can be interpreted equally well as motion in a straight line with an eye movement or as motion on a curved path (Figure 2). Instantaneously, the translation and rotation components of the two stimuli are identical. Although the displays diverge over time, Royden (1994) showed that this divergence is very small for the short durations used in previous experiments, and surmised that the difference is unlikely to be detectable by the visual system. Furthermore, the results of Royden and colleagues (Royden et al., 1992; Royden et al., 1994) in the simulated eye-movement condition were consistent with the idea that observers were perceiving a curved path and responding according to a point that corresponded with a future position on this path (Royden, 1994).

Royden (1994) pointed out that extraretinal signals concerning eye movements could disambiguate this stimulus.
In the real eye-movement condition, the extraretinal eye movement signals would indicate that the rotation was generated by an eye movement, so that the effects of the rotation in the visual image could be discounted, and observers would make accurate heading judgments. In the simulated eye-movement condition, the extraretinal signal would indicate that no eye movement was occurring, so the visual system would interpret the rotation as integral to the path of motion, and observers would perceive curved path motion. The ability to perceive curved path motion indicates that the visual system is able to compute both the translational and rotational components of observer motion from the visual input alone, without using eye movement information for computing rotation, because both translation and rotation, or a combination of the two, must be computed to estimate curved path motion. Therefore, if this information is available for judging curved paths from visual input alone, presumably it could be used to judge a straight line heading with an eye movement. This fact could explain why some experimental results showed that observers could judge their translational heading in the simulated eye-movement condition. However, it does not explain why some experiments lead to one set of results, the curved path interpretation, and others lead to another set of results, the straight line interpretation of the simulated eye-movement condition.

Recently, Li and Warren (2000) examined some factors that might help people disambiguate the curved path from the straight line interpretation of the simulated eyemovement condition. Rather than using displays consisting of random dots that moved to simulate observer motion toward a simulated scene, they used a densely textured ground plane. They also tested stimuli consisting of a textured ground plane on which there were either vertical posts or rectangular objects ("tombstones"). Li and Warren argued that the dense motion parallax provided by the textured ground plane would help in computing translation. They also claimed that the addition of well-defined objects could resolve the path ambiguity by allowing subjects to track a single object over time, something that would be difficult to accomplish with random-dot displays. They found that subjects could reliably judge their translational direction of motion with these textured displays and that performance improved with the addition of posts or tombstones. In a subsequent study (Li \& Warren, 2002), they showed that subjects could also steer accurately using this retinal information, provided the stimulus contained multiple reference objects.

In a more recent study, Li and Warren (2004) showed that the instructions given to the observer could influence the path perception. They tested observer heading perception under three conditions. In the neutral condition, subjects were told nothing about their path. In the curved path condition, they were told they were moving on a curved path. In the straight path condition, they were told they were moving on a straight path. Li and Warren found that observers showed large heading errors in the curved path condition, consistent with the perception that they were moving on a curved path. Subjects showed much 
A

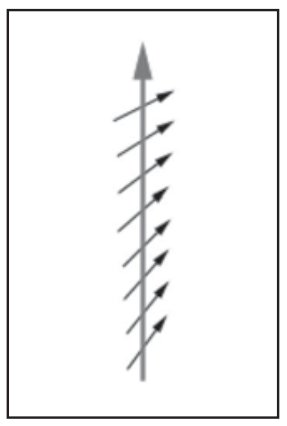

B

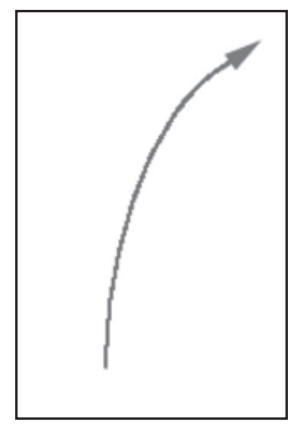

C

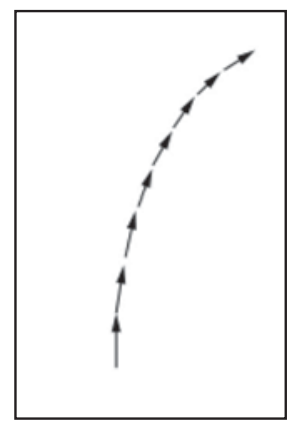

Figure 2. Schematic drawings of different observer paths. (A) Schematic of the path of an observer moving in a straight line while making an eye movement to fixate on a point in the scene. The gray arrow indicates the direction of observer motion. The black arrows indicate the direction of the observer's gaze. (B) Schematic of the perceived path of motion when the eye movement is simulated. (C) Schematic of the actual curved path of motion with the observer moving in the direction of his or her gaze, which is shifting over time.

more accurate heading judgments in both the neutral and straight path conditions, indicating that they judged their translation more accurately in these conditions. Li and Warren (2004) concluded that instructions do affect the perception of path when subjects are given this ambiguous stimulus, and that the default perception (when subjects were given no prior instructions) is consistent with the straight path interpretation of the stimulus.

Several questions are left unanswered by Li and Warren's studies. In their first study (Li \& Warren, 2000), they found that subjects showed large heading errors when the stimulus consisted of a random-dot field, as opposed to a textured ground plane with reference objects. This result led $\mathrm{Li}$ and Warren to conclude that dense motion parallax is important for computing translation. However, in their later study (Li \& Warren, 2004), subjects performed nearly as well with the random-dot displays as with the textured displays. They also showed that increasing dot density did not have any effect on the accuracy of heading judgments, leading them to conclude that dense motion parallax was not required for accurate heading perception. They suggested that the difference in the two studies arose from the difference in the size of the field of view used. In the original study, they used a small field of view for the random-dot stimulus $\left(69^{\circ} \times 59^{\circ}\right)$, whereas in the later study, they used a larger field of view $\left(112^{\circ} \times 95^{\circ}\right)$. Koenderink and van Doorn (1987) have shown that increasing the field of view provides more information for estimation of rotation from the optic flow field, thus allowing for more accurate heading judgments. Grigo and Lappe (1999) showed that a large field of view $\left(90^{\circ} \times 90^{\circ}\right)$ allowed observers to judge their heading accurately when approaching a frontoparallel plane with both translational and rotational motion. Thus, the idea that a larger field of view allows more accurate heading judgments is a plausible one, but it remains to be tested systematically.

In addition to the size of the field of view, another factor that could affect an observer's ability to judge his or her heading is the duration of the stimulus. In examining the differences between the straight path stimulus with a simulated eye movement and the curved path stimulus, it is clear that the two diverge over time, with the largest differences occurring at the edges of the stimulus (at the largest eccentricities) (Royden, 1994). Thus, increasing the field of view and/or extending the duration of the stimulus will reveal larger disparities between the two stimuli, perhaps allowing the visual system to disambiguate them.

In this study, we examined several factors that might lead observers to be able to disambiguate the curved path from the straight line interpretation of the simulated eye-movement condition. These included field of view, duration, and random-dot versus textured ground plane display with reference objects (tombstones). We also tested whether subjects could be influenced in their interpretation of the stimulus by explicit instructions given by the experimenter describing the path of motion, using a method similar to that of Li and Warren (2004) in their experiments. If the visual system is able to interpret the stimulus as either the straight path or the curved path, then we should be able to influence observers' judgments by giving them explicit descriptions of their path. By testing each of these factors systematically, we aimed to determine which, if any, of them influence an observer's path perception when given an ambiguous stimulus that can be interpreted as either a curved or straight path.

\section{GENERAL METHOD}

Observers viewed a simulated scene depicting motion over a ground plane, which consisted of either random dots or a textured plane with 45 rectangular objects (tombstones). For the small field of view experiments, the simulation was generated by a Macintosh G3 computer using a 17-in. CRT monitor with a frame rate of $75 \mathrm{~Hz}$. The observers were positioned $35 \mathrm{~cm}$ from the screen, resulting in a field of view of $51^{\circ} \times 39^{\circ}$. For the large 
field of view experiments, the simulation was generated by a Dell PC running Windows 98 and using a Sharp LCD overhead projector with a $75-\mathrm{Hz}$ frame rate. The observers sat $1 \mathrm{~m}$ from the screen for a field of view of $94^{\circ} \times$ $77^{\circ}$. Both sets of experiments used a display resolution of $600 \times 800$ pixels. All experiments were run in a darkened room.

Two different scenes were tested. One scene, similar to the scene used by Li and Warren (2000), consisted of a textured ground plane with 45 rectangular objects (tombstones) randomly positioned on the plane (Figure 3A). The texture consisted of a randomly generated Julesz pattern - that is, a grid of squares, each colored with a randomly chosen shade of gray. This texture was applied to both the ground plane and the tombstones. The texture was antialiased and mipmapped. The textured ground plane extended 2,000 m in every direction from the observer's initial location. The simulated eye height of the observer was $1.8 \mathrm{~m}$ above the

\section{A}

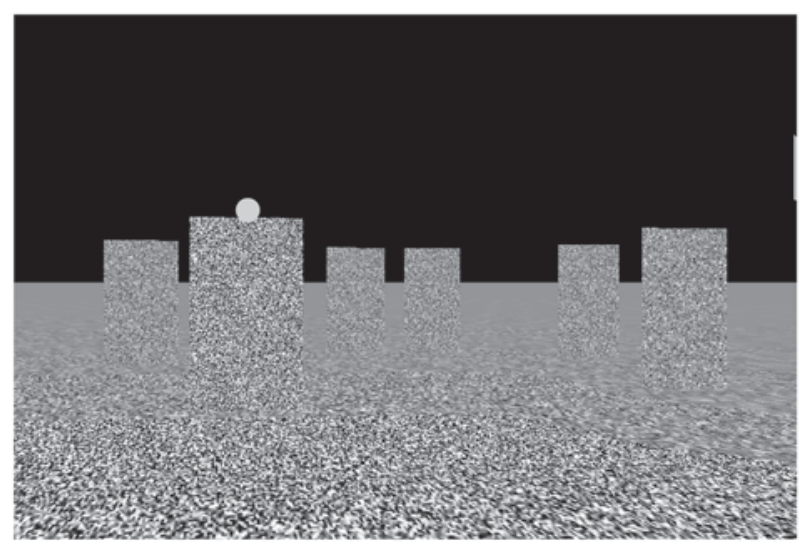

B

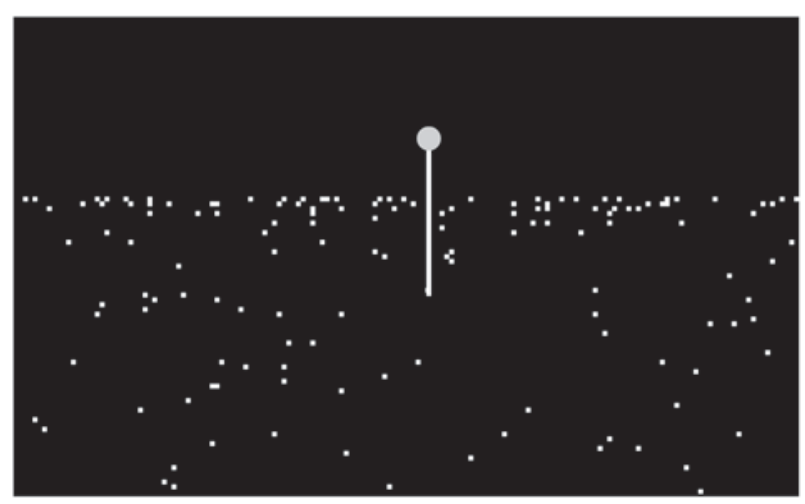

Figure 3. Simulated scenes used in this experiment. (A) The textured ground plane with rectangular tombstones. Only a portion of the scene is shown. The gray circle shows how the fixation point was affixed to a tombstone. In the experiments, the fixation point was colored red. (B) The random-dot stimulus. The stimulus consisted of random dots distributed on a ground plane. Only a portion of the scene is shown. The gray circle on the white line shows the positioning of the fixation point attached to the ground plane. In the experiments, the fixation point was red. ground plane. The tombstones measured $1.0 \mathrm{~m}$ in width and $1.8 \mathrm{~m}$ in height. The fixation point was located at the top of a slightly taller tombstone $(2.1 \mathrm{~m})$, so that it would not be occluded by other tombstones during the trial. The random-dot scene consisted of 5,000 dots randomly positioned on a ground plane extending $10 \mathrm{~m}$ in depth (Figure 3B). The plane was a triangular shape. At a given distance, $z$, from the observer, the width of the plane was $4 z$. This was wide enough that the edge of the plane did not appear on the screen as the observer rotated. To ensure that the dots were not too sparse at nearby locations, half of the dots were placed between 0 and $1 \mathrm{~m}$ and half were placed between $0.5 \mathrm{~m}$ and $10 \mathrm{~m}$. Each dot consisted of a single pixel, measuring $0.12^{\circ} \times 0.13^{\circ}$ in the large field of view display. The fixation point was attached to the ground plane by a thin line that was the same height as the fixation tombstone in the textured scene.

During a trial, the image moved to simulate observer translation forward with a speed of $200 \mathrm{~cm} / \mathrm{sec}$ and a translation direction chosen at random between $0^{\circ}, \pm 5^{\circ}$, and $\pm 10^{\circ}$ from the center of the screen. Rotation rates of $0^{\circ}, 2^{\circ}, 5^{\circ}$, or $8^{\circ} / \mathrm{sec}$ to the left or right were tested. Because the fixation point was attached to the scene, the rotation rate varied over the course of the trial. The rotation rate for each trial was calculated as the mean rotation rate during the trial, as described in Li and Warren (2000). To obtain the desired mean rotation rate, the initial position of the fixation point was computed in terms of its distance from the observer and its initial angle from the simulated heading direction. Thus, for the mean rotation rates of $\pm 0^{\circ}$, $2^{\circ}, 5^{\circ}$, and $8^{\circ} / \mathrm{sec}$, the initial angles were $\pm 0^{\circ}, 7^{\circ}, 10.2^{\circ}$, and $17^{\circ}$, and the initial distances were $10,10,7$, and $7 \mathrm{~m}$, respectively. For the faster rotation rates, smaller initial distances were required so that the fixation point would remain on the screen over the course of the trial.

There were two main conditions for the rotation. In the real eye-movement condition, rotation was generated by a real eye movement of the observer. The observer tracked a fixation point that was attached to the ground plane by a vertical pole (in the dot scenes) or by one of the tombstones (in the textured scene). In the simulated eyemovement condition, observers fixated a stationary point attached to the ground plane by a pole or tombstone, as described above. The dots or texture moved to simulate the motion that would occur if the observer were translating while making an eye movement. At the end of each trial, observers used a cursor to position a probe at the location that coincided with their perceived direction of motion. The probe was a line approximately $2.5 \mathrm{~m}\left(15^{\circ}\right)$ high, attached to the ground plane at a distance of $7.5 \mathrm{~m}$ from the observer.

All observers tested were naive to the hypotheses being tested in these experiments, except for one observer who worked in the lab and may have been aware of the hypotheses. This observer participated in all the experiments except for the random-dot condition in Experiment 2.

We varied several parameters to examine their effects on observer responses. In Experiment 1, we examined the effect of the size of the field of view. In Experiment 2, we 
increased the duration of the stimulus, and compared responses to a random-dot stimulus with responses to a textured scene containing reference objects. In Experiment 3, we examined the effect of explicit path description on the observers' responses.

\section{EXPERIMENT 1}

Li and Warren (2000) reported that using a textured scene rather than random dots gave observers the ability to judge their straight line motion in the simulated eyemovement condition. The addition of reference objects further increased observers' accuracy in this task. The use in their experiment of a fairly large field of view, $112^{\circ} \times$ $95^{\circ}$, could also have contributed to observers' abilities to judge their translational motion in this condition. In the present experiment, we tested observers' abilities to judge their path of motion for both a small $\left(51^{\circ} \times 39^{\circ}\right)$ and a large $\left(94^{\circ} \times 77^{\circ}\right)$ field of view.

\section{Method \\ In this experiment, both conditions consisted of the textured ground plane with 45 tombstones positioned at random on the plane, as described in the General Method section. The observers viewed the display on a 17 -in. CRT monitor in the small field of view $\left(51^{\circ} \times\right.$ $39^{\circ}$ ) condition and on a large screen, with the scene projected from an overhead projector, for the large field of view $\left(94^{\circ} \times 77^{\circ}\right)$ condi- tion. The duration of each trial was $1.0 \mathrm{sec}$. In each block of trials, six rotation rates were presented for each of the two eye movement condi- tions. A condition with no rotation was also presented, for a total of 13 conditions. The conditions were randomly interleaved, with each condition presented 10 times, giving a total of 130 trials. The heading was randomly chosen to be $0^{\circ}, \pm 5^{\circ}$, and $\pm 10^{\circ}$ from the center of the screen. The rotation rates tested were $0^{\circ}, \pm 2^{\circ}, \pm 5^{\circ}$, and $\pm 8^{\circ} / \mathrm{sec}$ in all cases except for the small screen, real eye-movement condition, in which the rotation rates tested were $0^{\circ}, \pm 2^{\circ}, \pm 5^{\circ},-7^{\circ}$, and $-8^{\circ} / \mathrm{sec}$. Seven observers participated in each of the small screen and large screen experiments, and 3 observers participated in both conditions.}

\section{Results}

Figure 4 shows the results for Experiment 1. The circles show the results with the small field of view and the squares indicate the results with the large field of view. In both cases, the observers judged their heading accurately in the real eye-movement condition (represented by filled symbols), with the maximum error being $6.4^{\circ}$ (average of $2.5^{\circ}$ ) for the small screen and $2.3^{\circ}$ (average of $1.1^{\circ}$ ) for the large screen. In contrast, in the simulated eye-movement condition (represented by open symbols) there was a bias in the direction of the simulated rotation that increased with the increasing rotation rate, with a maximum error of $15.8^{\circ}$ in the small screen and $12.3^{\circ}$ in the large screen conditions. This bias is consistent with a curved path interpretation of the stimulus.

Two-way ANOVA with rotation rate and field of view as factors showed no significant main effect of field of view in either the real eye-movement case $[F(1,83)=0.66$, n.s. $]$ or the simulated eye-movement case $[F(1,83)=0.32$, n.s.]. There was a significant main effect of rotation rate in both the real $[F(5,83)=2.86, p<.05]$ and simulated $[F(5,83)=48.65, p<.0001]$ conditions. There was no

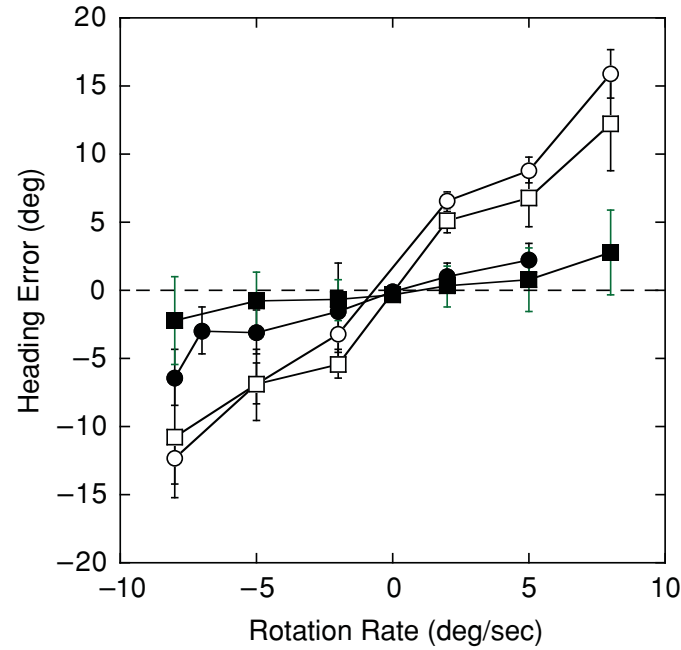

Figure 4. Results of Experiment 1. Each symbol represents the heading error averaged over all the subjects in each condition of the experiment. Open symbols indicate responses in the simulated eye-movement condition, and filled symbols indicate responses in the real eye-movement condition. Circles and squares show the responses for the small field of view and the large field of view, respectively. Error bars are \pm 1 standard error.

significant interaction between rotation rate and field of view in either condition [real, $F(5,83)=0.75$, n.s.; simulated, $F(5,83)=1.34$, n.s.]. Thus, although the maximum error was slightly less in the large field of view condition, this decrease was not significant. Much of the decrease may have been due to a single observer, who showed a bias in the direction opposite the rotation rate (and thus in a direction opposite that for all the other observers) in the large field of view condition.

\section{EXPERIMENT 2}

The results of Experiment 1 are surprising, because they did not replicate the results of Li and Warren (2000, 2004). Unlike Li and Warren's subjects, our subjects showed a significant bias in the simulated eye-movement condition for a textured scene containing reference objects, in both a small and a large field of view. One factor that may have affected these results was the duration of the stimulus. In Experiment 1, we used a 1.0-sec-duration stimulus, whereas Li and Warren used a 1.5-sec-duration stimulus. In Experiment 2, we increased the duration of the stimulus to $1.5 \mathrm{sec}$. We also tested how well people judged heading with a scene consisting of random dots, so we could compare the results with those for the textured scene with reference objects.

\section{Method}

The methods for this experiment were identical to those used for the large field of view in Experiment 1, except that the duration was increased to $1.5 \mathrm{sec}$ and we ran experiments to test both the textured scene with reference objects and a random-dot scene (as described in the General Method section). Five subjects participated 
in the textured-scene condition and 5 subjects participated in the random-dot experiment. In each case, 2 observers had participated in Experiment 1. One observer participated in both conditions in Experiment 2.

\section{Results}

The results of Experiment 2 (Figure 5) indicate that increasing the duration of the stimulus to $1.5 \mathrm{sec}$ does not significantly affect the results for the simulated eye-movement conditions. For the textured scene, observers were very accurate for the real eye-movement condition, shown with the filled circles (maximum error of $4.8^{\circ}$ ). These results were not significantly better than the results for the large field of view, 1.0-sec duration shown in Experiment 1, because there was no main effect of duration $[F(1,69)=0.01$, n.s.], and no interaction between duration and rotation rate $[F(6,69)=0.68, \mathrm{n} . \mathrm{s}]$. There was also no significant main effect of rotation rate $[F(6,69)=0.73$, n.s. $]$.

In the simulated eye-movement condition with the textured scene, represented by the open circles, observers again showed an increased bias with increased rotation rate (maximum error of $17.6^{\circ}$ ), consistent with the perception of the curved path. This error was slightly larger than the error seen for the shorter duration in the large field of view in Experiment 1. A two-way ANOVA showed a significant main effect of rotation $[F(5,59)=22.72, p<$ $.0001]$. There was no main effect of duration $[F(1,59)=$ $0.23, \mathrm{n} . \mathrm{s}]$, but the interaction between the rotation rate and the duration barely reached the .05 level of significance $[F(5,59)=2.42, p<.05]$. It is likely that most of this difference was caused by the 1 observer in Experiment 1 who showed a bias in the direction opposite that shown by everyone else.

For the random-dot display, errors for the real eyemovement condition are shown by the filled squares.

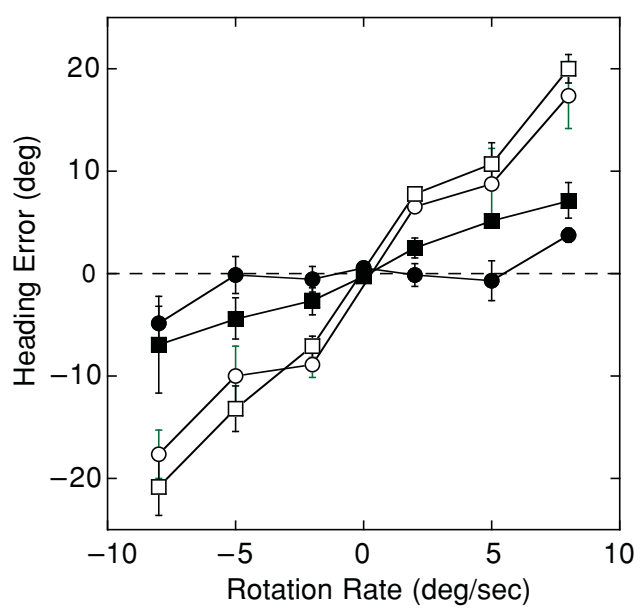

Figure 5. Results of Experiment 2. Each symbol represents the average heading error for all subjects. Filled and open symbols indicate results for the real and simulated eye-movement conditions, respectively. Squares show results for the random-dot scene and circles show the results for the textured scene. Error bars are \pm 1 standard error.
Errors in this condition (maximum error of $7.1^{\circ}$ ) were somewhat larger than in the results using the textured scene. Two-way ANOVA showed a main effect for rotation rate $[F(6,69)=13.04, p<.0001]$. There was no main effect of scene type $[F(1,69)=0.23$, n.s.], but there was a significant interaction between scene type and rotation rate $[F(6,69)=3.31, p<.01]$. In the simulated eye-movement condition (shown by open squares), errors were slightly higher than for the textured scene (maximum of $20.8^{\circ}$ ), but this difference was not statistically significant. Whereas there was a significant main effect of rotation rate $[F(6,59)=88.02, p<.0001]$, there was no main effect of scene type $[F(1,59)=0.02$, n.s. $]$ and no significant interaction between scene type and rotation rate $[F(1,59)=0.70$, n.s. $]$. Thus, in the simulated eyemovement condition, we found little difference between the results for the textured and random-dot scenes.

\section{EXPERIMENT 3}

Experiments 1 and 2 showed that adding texture and reference objects and increasing the field of view and the duration of the stimulus had no significant effect on observers' judgments in the simulated eye-movement condition. This leads to the question of whether observers could judge their translational direction of motion at all, under these conditions. In Experiment 3, we sought to address this question by giving observers an explicit description of the path on which they were moving.

\section{Method}

This experiment was identical to Experiment 2, except the observers were given an explicit description of their path of motion before the experiment started. In Experiment 2, the observers were not told anything about the shape of the simulated path of motion, they were merely asked to indicate where they were going in the display. In Experiment 3, the observers were told that they were moving in a straight line while rotating. They were explicitly told that the motion is "as if you are sitting on a swivel chair that is spinning as it moves forward in a straight line." All other parameters were the same as for Experiment 2. We tested both the random-dot stimulus and the stimulus with a textured ground plane with tombstones. Six observers participated in the experiment with the textured surfaces and 5 observers participated in the experiment with random dots. All but 2 of the observers had participated in at least one condition of the previous experiments. One observer participated in both conditions of Experiment 3.

\section{Results}

Figure 6 shows the results of Experiment 3. For both the textured scene (Figure 6A) and the random-dot scene (Figure 6B), observers still judged heading well in the real eye-movement condition (shown with filled squares), with a maximum error of $1.1^{\circ}$ in the textured condition and a maximum error of $2.4^{\circ}$ in the dot condition. For the textured scene, a two-way ANOVA for the real eye-movement condition showed no significant effect of rotation rate $[F(6,69)=1.11, \mathrm{n} . \mathrm{s}]$ or instructions $[F(1,69)=0.05$, n.s. $]$ and no interaction between the two $[F(6,69)=0.84$, n.s. $]$. For the random-dot scene with real eye movements, there is a significant main effect of rotation rate $[F(6,69)=$ 

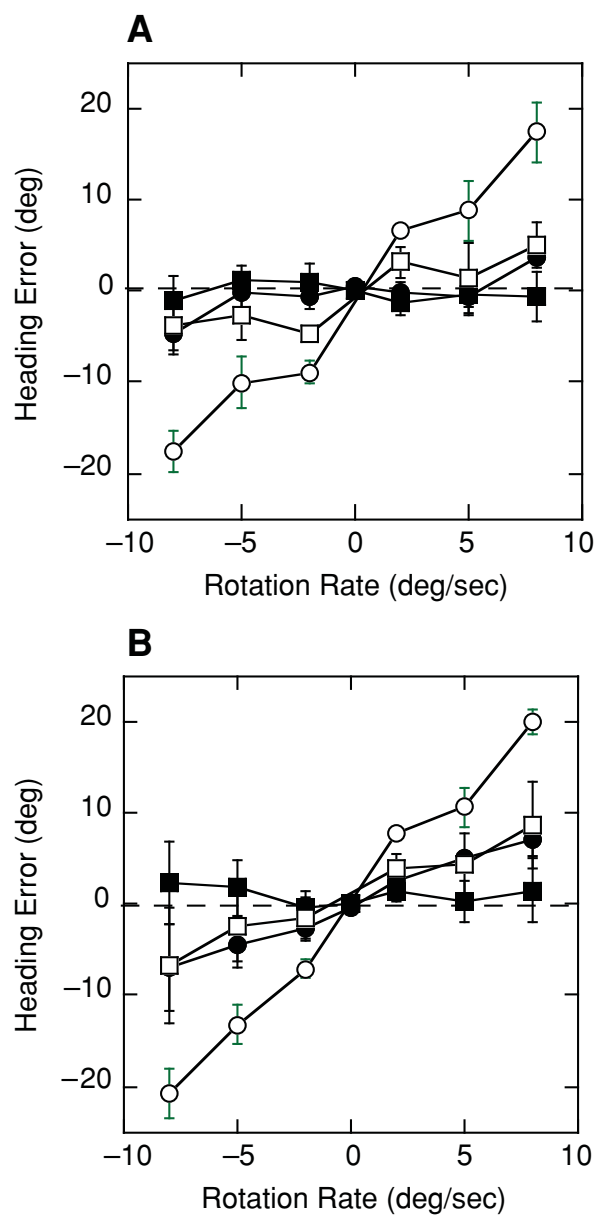

Figure 6. Results of Experiment 3. Each symbol represents the heading error averaged over all subjects. Filled and open symbols indicate results for the real and simulated eye-movement conditions, respectively. Circles show the results from Experiment 2, with no path description. Squares show the results from Experiment 3 , in which observers were given an explicit description of their path of motion. Error bars indicate \pm 1 standard error. (A) Results for the textured scene. (B) Results for the random-dot stimulus.

$2.56, p<.05]$. There was no significant effect of instructions $[F(1,69)=0.64$, n.s. $]$, but there was a significant interaction between the rotation rate and instruction condition $[F(6,69)=3.20, p<.01]$. The significant interaction reflects the increase in accuracy seen in the condition in which subjects were given an explicit path description in the real eye-movement condition.

In the simulated eye-movement condition, observers were considerably more accurate when given an explicit description of the path of motion (represented by open squares) for both scenes when compared with the previous experiments (e.g., Experiment 2, shown with open circles). In the textured scene condition, the maximum error for the simulated eye-movement case was $5.0^{\circ}$, considerably smaller than $17.6^{\circ}$, which was the result of Experiment 2, without instructions. An ANOVA showed a significant effect of rotation rate $[F(5,59)=18.12, p<$ $.0001]$. Whereas there was no significant main effect of instructions $[F(1,59)=0.08$, n.s.], there was a significant interaction between rotation rate and the instruction condition $[F(5,59)=8.24, p<.0001]$. This interaction reflected the large decrease in error seen for the condition in which subjects were given an explicit description of the path. Similarly, with the random-dot display, observers performed with much greater accuracy in the simulated eye-movement condition when given an explicit description of the path (maximum error of $8.7^{\circ}$ ) than they did in Experiment 2, with no description (maximum error of $20.8^{\circ}$ ). As with the textured scene, there was a significant effect of rotation rate $[F(5,59)=21.87, p<.0001]$. There was not a significant main effect of instructions $[F(1,59)=0.69$, n.s. $]$, but there was a significant interaction between rotation and instructions $[F(5,59)=4.98$, $p<.001]$, reflecting the decrease in slope seen with the condition with explicit path description.

There was no significant difference between the results for the dot scene and the textured scene for this condition with instructions. In the real eye-movement condition, a two-way ANOVA showed no significant main effect of rotation rate $[F(6,69)=0.08$, n.s. $]$ or scene type $[F(1,69)=$ 0.60 , n.s. $]$, and there was no significant interaction between the two $[F(6,69)=0.22$, n.s. $]$. In the simulated eyemovement condition, there was a significant main effect of rotation rate $[F(5,59)=2.74, p<.05]$, but no significant effect of scene type $[F(1,59)=0.38$, n.s. $]$ and no significant interaction between the two $[F(5,59)=0.50$, n.s.]. Thus, as was the case in Experiment 2, observers performed similarly for both the random-dot scene and the textured scene.

\section{DISCUSSION}

The results of Experiments 1 and 2 suggest that observers' heading judgments are consistent under a wide variety of conditions, including small and large field of view, scenes composed of random dots or textured surfaces with reference objects, and durations of 1.0 or $1.5 \mathrm{sec}$. In all these conditions, observers judged their translational heading well in the real eye-movement condition, but showed a bias in heading judgments that increased with increasing rotation rates for the simulated eye-movement condition. These results are consistent with the original results of Royden and colleagues (Royden et al., 1992; Royden et al., 1994) and with the idea that observers perceive the simulated eye-movement condition as motion on a curved path (Royden, 1994).

Interestingly, we did not replicate the results of $\mathrm{Li}$ and Warren (2000), who found that observers gave accurate heading judgments in the simulated eye-movement condition when presented with a large field of view with a textured plane and reference objects. Our results suggest that, with a large field of view in the simulated eye-movement condition, there is no significant difference between a random-dot stimulus and the textured ground plane with reference objects. For both scenes, our subjects responded similarly, showing large biases in the simulated 
eye-movement condition. This weighs against the hypothesis submitted by Li and Warren (2000) that dense motion parallax and the ability to track individual objects lead to a disambiguation of the two possible perceptions of the simulated eye-movement condition. This is a result similar to that of Li and Warren (2004), who found little difference between observer responses for random-dot scenes and textured scenes or those with multiple reference objects. However, our results differ from those of $\mathrm{Li}$ and Warren (2004) with regard to the default perception of path. Li and Warren found that observers in the neutral condition, with no instructions regarding path, gave responses consistent with the straight path perception. In the present study, when given no explicit description of the path they would travel on, all of our subjects responded in a way that was consistent with the perception of a curved path, rather than the straight line interpretation that was the actual stimulus.

It is not clear why our results differ from those of $\mathrm{Li}$ and Warren (2000) with respect to subjects' ability to judge straight path motion when given a textured scene with multiple reference objects. However, the results of Experiment 3 and the results of Li and Warren (2004) could shed some light on this topic. In our experiments, as with those of Li and Warren, when given an explicit description of the motion that was being simulated (i.e., that of moving in a straight line while rotating, as if they were in a swivel chair) our observers, on average, were able to judge their direction of translational motion accurately both for the random-dot scene and for the textured scene. This result implies that the visual system is able to use visual input alone to compute the translational component of motion, independently of the rotational component. It further shows that people have access to this information, allowing them to make these straight line judgments when they know in advance what type of translation and rotation motion is occurring. Thus, the difference in the results of our Experiments 1 and 2 and those of $\mathrm{Li}$ and Warren (2000) could have been caused by a subtle difference in the way observers were instructed to give their responses or by a difference in the expectations of the observers in the two experiments. In our studies, observers seemed to show a strong bias toward the curved path interpretation. Without an explicit path description, all but one subject gave responses consistent with the curved path interpretation. Even with explicit instructions describing a straight path of motion, some individuals still gave responses consistent with a curved path.

Li and Warren (2004) concluded that instructions can influence the perception of this stimulus, but that the default perception for the large field of view is that of motion on a straight line. Our findings that instructions can influence the perception of the stimulus agree with theirs, but differ in that the default perception for a large field of view in our experiments appears to be the curved path. Some possible explanations for this difference could be difference in the order of presentation, difference in dot density for the random-dot display, or difference in the size of the large field of view. The order of presentation of the stimuli differed between our experiments and those of Li and Warren (2004). We always ran the experiments with no instructions (similar to Li and Warren's "neutral" case) before we ran the experiments with the explicit path description, so that the observers in the neutral case would not be influenced by previous path descriptions. $\mathrm{Li}$ and Warren randomized the order, so that some of the observers in the neutral case would have been given the straight line description in a previous condition and some would have been given a curved path description. It seems plausible that these previous conditions could have influenced the observers' responses in the neutral case, and that some observers would thus tend to see more of a curved path and others more of a straight line, depending on what they had been told previously. This would lead to a result somewhat in between the two conditions, as seen in $\mathrm{Li}$ and Warren's data for Experiment 1. This explanation can only account for part of the difference seen, and does not account for the accurate judgments observed in their second experiment.

Another difference between our random-dot stimulus and that of Li and Warren (2004) is the density of dots. Their stimulus used a uniform distribution of dots, giving a very sparse projection for the nearby locations. They tested two different densities and found no effect of dot density for either the straight or curved path instructions. Our stimuli used a higher density overall, and we used a nonuniform distribution, with a higher dot density (in the simulated world) for nearby locations. It is possible that this difference led to the differing results for the random-dot cases. However, we feel this is unlikely, for two reasons. First, as mentioned above, Li and Warren (2004) found no effect of dot density. Second, the difference between our results and those of $\mathrm{Li}$ and Warren (2004) goes in the opposite direction from what one would expect. Increasing the dot density should increase the motion parallax information needed to eliminate rotation and compute a straight path of motion. However, we found that the default path for our random-dot stimulus was a curved path, and observers did not perceive a straight path unless explicitly instructed that their path was straight. It seems unlikely that increasing dot density would lead observers to favor the curved path over the straight path interpretation. In addition, a difference in dot density does not account for the difference in results between our experiments and those of Li and Warren $(2000,2004)$ in the case of the textured plane with reference objects.

A third possible explanation for the difference in results could be the influence of field of view. As previously discussed, a large field of view provides more information for computing both rotation and translation components of observer motion (Koenderink \& van Doorn, 1987). The large field of view used in our experiments $\left(94^{\circ} \times 77^{\circ}\right)$ was not as large as that used by Li and Warren (2004) $\left(112^{\circ} \times 95^{\circ}\right)$. This difference could have led to the differing default perception of a curved path as opposed to a straight path. However, if field of view were the primary factor causing this difference, one would expect to see some discrepancy in our results between the small field 
of view $\left(51^{\circ} \times 39^{\circ}\right)$ and the larger field of view, which is more than 3.5 times the size in area. Our results showed no significant difference between these two. So, although it is possible that a further increase (by a factor of about 1.5 ) in the area of the field of view could cause the default perception to flip from curved path to straight path, it seems unlikely.

Although we did not test a condition in which we explicitly described a curved path, the results from Experiments 1 and 2 show errors of similar magnitude to those seen by Li and Warren (2004) when they explicitly told observers that they were moving on a curved path. Thus, we would expect that giving observers an explicit description of a curved path would yield results similar to those in Experiment 2.

Given the results of our Experiment 3 and the results of $\mathrm{Li}$ and Warren (2004) with regard to the effect of instructions, it seems most likely that the difference in the default path perception is primarily due to subtle differences in experimental setup and subject expectations. The combined results of the two studies suggest that neither interpretation is dominant under the conditions tested, but rather, that the interpretation depends on the expectations of the subjects.

\section{Resolving Conflicting Results}

Over the last decade, there has been considerable debate in the literature about whether humans can judge from visual information alone the translational component of their motion in the presence of rotations. Some experimental results, in which observers showed increasing bias with increased rotation rates in the simulated eye-movement condition, suggested that observers needed extraretinal information to compute this translational component (Banks et al., 1996; Ehrlich et al., 1998; Royden et al., 1992; Royden et al., 1994). Results from other labs, indicating accurate heading judgments in the simulated eyemovement case, suggested that observers could compute translational motion from visual input alone (van den Berg, 1992, 1993, 1996; Warren \& Hannon, 1988, 1990). The results presented here suggest that under the right conditions, people can interpret the stimulus shown in the simulated eye-movement condition as either motion on a curved path or motion along a straight line while making an eye movement. In the present experiments, the default interpretation of the stimulus used in the simulated eyemovement condition appears to be that of motion on a curved path. This is consistent with the results from other experiments (Banks et al., 1996; Ehrlich et al., 1998; Royden et al., 1992; Royden et al., 1994). However, when given an explicit description of the path, observers were able to judge their translational direction fairly accurately, consistent with the reports of other researchers (van den Berg, 1992, 1993, 1996; Warren \& Hannon, 1988, 1990). Thus, we are able to replicate both sets of seemingly conflicting results. This supports the idea that the stimulus used in the simulated eye-movement condition is an ambiguous one that can be interpreted as motion on a curved path or motion in a straight line with an eye movement. Although it is unclear why the results in different labs differ with regard to whether observers respond according to the curved path or straight line interpretation, the results here suggest that the discrepancies could be due to subtle differences in how observers were instructed or in the expectations or previous experience of the observers participating in the experiment.

Whereas our experiment and that of Li and Warren (2004) examined the effect of explicitly describing the simulated path of motion, van den Berg (1996) has presented evidence that other shifts in a subject's attention may also affect the perceived path. Van den Berg noticed that there is an illusory motion in depth of the stationary fixation point in the simulated rotation displays. When instructed to make heading judgments based on this illusory motion of the fixation point, observers judged heading accurately, consistent with a perceived straight path of motion. When instructed to make judgments based on the entire motion pattern, observers showed large errors, consistent with the curved path perception. Thus, other manipulations of the subjects' expectations or strategies for judging heading appear to alter the perception between the curved and straight path interpretations. This result adds weight to the idea that the differing results between labs, including the differences between the default path perception, are likely due to subtle differences in subject expectations and strategies.

One question that has not been addressed is the exact curvature of the path that observers perceive in the curved path condition. Both the present study and previous studies have measured the perceived destination point under various conditions, but have not attempted to measure the actual perceived path and its curvature. Warren and colleagues (Warren, Mestre, Blackwell, \& Morris, 1991) have shown that people can judge the curvature of their path well under certain conditions. An interesting topic for future research might be whether the perceived curvature would change under some of the different conditions tested here (i.e., increased field of view, texture with and without reference objects, etc.).

Our results, along with those of Li and Warren (2004), support the idea that the visual system can compute the translational component of observer motion from visual information alone, even in the presence of fairly large rotations. This ability does not require a textured scene or multiple identifiable objects, as seen in the results of Experiment 3, in the random-dot case, in which there was only a single reference object. In our experiments, in contrast to those of Li and Warren (2004), this interpretation was not automatic; observers had to be told explicitly that their path of motion was on a straight line. Although extraretinal information does not appear to be required for observers to discount the rotational component of their motion, it does appear to help disambiguate the visual stimulus, leading observers to judge the stimulus reliably as straight line motion with an eye movement, independent of other variables in the display. In the real eye-movement condition, observers did not need to be instructed that they were moving in a straight line in order to indicate accurately their translational direction of motion. 


\section{REFERENCES}

Banks, M. S., Ehrlich, S. M., Backus, B. T., \& Crowell, J. A. (1996). Estimating heading during real and simulated eye movements. Vision Research, 36, 431-443.

Beintema, J. A., \& VAN den Berg, A. V. (1998). Heading detection using motion templates and eye velocity gain fields. Vision Research, 38, 2155-2179.

Cutting, J. E., Springer, K., Braren, P. A., \& Johnson, S. H. (1992). Wayfinding on foot from information in retinal, not optical, flow. Journal of Experimental Psychology: General, 121, 41-72.

Ehrlich, S. M., Beck, D. M., Crowell, J. A., Freeman, T. C. A., \& BANKS, M. S. (1998). Depth information and perceived self-motion during simulated gaze rotations. Vision Research, 38, 3129-3145.

Gibson, J. J. (1950). The perception of the visual world. Boston: Houghton Mifflin.

Gibson, J. J. (1966). The senses considered as perceptual systems. Boston: Houghton Mifflin.

Grigo, A., \& LAPPE, M. (1999). Dynamical use of different sources of information in heading judgments from retinal flow. Journal of the Optical Society of America A, 16, 2079-2091.

Hatsopoulos, N. G., \& WarReN, W. H. (1991). Visual navigation with a neural network. Neural Networks, 4, 303-317.

Heeger, D. J., \& Jepson, A. D. (1992). Subspace methods for recovering rigid motion I: Algorithm and implementation. International Journal of Computer Vision, 7, 95-117.

HiLdRETH, E. C. (1992). Recovering heading for visually-guided navigation. Vision Research, 32, 1177-1192.

Koenderink, J. J., \& van Doorn, A. J. (1987). Facts on optic flow. Biological Cybernetics, 56, 247-254.

LAPPE, M., \& RAUSCHECKER, J. P. (1993). A neural network for the processing of optic flow from ego-motion in man and higher mammals. Neural Computation, 5, 374-391.

LI, L., \& Warren, W. H. (2000). Perception of heading during rotation: Sufficiency of dense motion parallax and reference objects. Vision Research, 40, 3873-3894.

LI, L., \& WARrEN, W. H. (2002). Retinal flow is sufficient for steering during observer rotation. Psychological Science, 13, 485-491.

LI, L., \& Warren, W. H. (2004). Path perception during rotation: Influence of instructions, depth range, and dot density. Vision Research, 44, 1879-1889.

Longuet-Higgins, H. C., \& PraZdny, K. (1980). The interpretation of a moving retinal image. Proceedings of the Royal Society of London: Series $B$, 208, 385-397.
Perrone, J. A. (1992). Model for the computation of self-motion in biological systems. Journal of the Optical Society of America A, 9, 177-194.

Perrone, J. A. \& Stone, L. S. (1994). A model of self-motion estimation within primate extrastriate visual cortex. Vision Research, 34, 2917-2938

Regan, D., \& Beverley, K. I. (1982). How do we avoid confounding the direction we are looking and the direction we are moving? Science, 215, 194-196.

Rieger, J. H., \& Lawton, D. T. (1985). Processing differential image motion. Journal of the Optical Society of America A, 2, 354-360.

Rieger, J. H., \& ToET, L. (1985). Human visual navigation in the presence of 3-D rotations. Biological Cybernetics, 52, 377-381.

ROYDEN, C. S. (1994). Analysis of misperceived observer motion during simulated eye rotations. Vision Research, 34, 3215-3222.

Royden, C. S. (1997). Mathematical analysis of motion-opponent mechanisms used in the determination of heading and depth. Journal of the Optical Society of America A, 14, 2128-2143.

Royden, C. S., Banks, M. S., \& Crowell, J. A. (1992). The perception of heading during eye movements. Nature, 360, 583-585.

Royden, C. S., Crowell, J. A., \& Banks, M. S. (1994). Estimating heading during eye movements. Vision Research, 34, 3197-3214.

Stone, L. S., \& Perrone, J. A. (1997). Human heading estimation during visually simulated curvilinear motion. Vision Research, 37, 573-590.

VAN DEN BERG, A. V. (1992). Robustness of perception of heading from optic flow. Vision Research, 32, 1285-1296.

van DEN Berg, A. V. (1993). Perception of heading. Nature, 365, 497 498.

VAN DEN BERG, A.V. (1996). Judgments of heading. Vision Research, 36, 2337-2350.

WARREN, W. H., \& HANNON, D. J. (1988). Direction of self-motion is perceived from optical flow. Nature, 336, 162-163.

WARREN, W. H., \& HANNON, D. J. (1990). Eye movements and optical flow. Journal of the Optical Society of America A, 7, 160-169.

Warren, W. H., Mestre, D. R., Blackwell, A. W., \& Morris, M. W. (1991). Perception of circular heading from optical flow. Journal of Experimental Psychology: Human Perception \& Performance, 17, 28-43.

(Manuscript received August 20, 2004; revision accepted for publication April 18, 2005.) 